\title{
One Day Polyethylene Glycol-3350 for Bowel Preparation in Pediatrics: A Literature Review
}

\author{
Shristi Shakya', Sumisti Shakya², Zhongyue Li $^{*}$ \\ ${ }^{1}$ Department of Gastroenterology, Children's Hospital of Chongqing Medical University, Chongqing, China \\ ${ }^{2}$ Department of Obstetrics and Gynaecology, The Second Affiliated Hospital of Chongqing Medical University, \\ Chongqing, China \\ Email: shristi shakya@hotmail.com, sumisti@hotmail.com, "lizhongyue1001@hotmail.com
}

Received 28 August 2015; accepted 20 September 2015; published 23 September 2015

Copyright (C) 2015 by authors and Scientific Research Publishing Inc.

This work is licensed under the Creative Commons Attribution International License (CC BY).

http://creativecommons.org/licenses/by/4.0/

(c) (7) Open Access

\begin{abstract}
Bowel preparation for colonoscopy in children is a challenging procedure. Wide variety of preparation protocols exist, varying with the hospital. Unlike in adults, there is a lack of uniform bowel preparation protocol in children. Ideally, the bowel preparation agents are assessed by their safety, efficacy and tolerability. Unfortunately, none of the preparations currently available meets all of these criteria. However, since last decade, Polyethylene Glycol-3350 (PEG-3350) is gaining popularity for bowel preparation with reported safety, efficacy, and tolerability. The only major drawback of PEG-3350 without electrolyte was 4 days long preparation time thus raising the question if the duration of preparation time could be minimized and yet have same efficacy, safety, and tolerability of the medicine. Hence, one day PEG-3350 regimen was introduced eventually and is now being studied with increased dosage or combined with other laxatives. This is the first review which compiles the study so far conducted on one day PEG-3350 without electrolyte as colonoscopy bowel preparation in children and tries to summaries if this regimen can be commonly used in children for colonoscopy bowel preparation.
\end{abstract}

\section{Keywords}

Bowel Preparation, One Day Preparation, Pediatrics, PEG-3350

\section{Introduction}

Bowel preparation for colonoscopy in children is a challenging procedure. Over the years, a wide variety of bo${ }^{*}$ Corresponding author. 
wel preparation regimens have been used in children [1]-[4]. Medication that has been used is high dose Polyethylene glycol (PEG) with electrolyte, which in published studies has shown high efficacy [5], but had poor palatability due to its distinctive unpleasant taste along with poor tolerability by children because of large volume that must be complete within a short period of time which frequently required nasogastric tube [2] [3] [6] [7]. Magnesium citrate, or combinations with stimulants had poor palatability and needed dietary restrictions [2] [8]. Oral sodium phosphate in children is limited because of serious adverse effects such as hyperphosphatemia, hypocalcemia [8]-[11], acute kidney injury [12] and can result in colonic mucosal changes that mimic inflammatory bowel disease [13]. Enemas alone or in combination with stimulants that required anal insertion [10]. Bisacodyl or senna alone required clear liquid diet for 2 - 3 days with multiple enemas before colonoscopies examination with still had high poor preparation rate requiring repeated examination [2].

Ideally bowel preparation agents are judged by their safety, efficacy and tolerability [14]-[16]. No bowel preparation regimen meets the ideal criteria for bowel cleansing [2]. Beside these three ideal criteria, the other aspects of bowel preparation in pediatrics are ease of administration, palatability, dietary restriction, and daily routine disruption minimization [1].

Since last decade, Polyethylene glycol-3350 (PEG-3350), an osmotic laxative is commonly used as bowel preparation and has recently gained popularity [6] [14] [15] [17] [18]. PEG-3350 as a bowel preparation in children was first reported by Pashanker et al. [14] with administration dose of $1.5 \mathrm{~g} / \mathrm{kg} /$ day for 4 days. It has shown to be effective and safe. Because of its tasteless character, it can be mixed with various types of drinks according to patient's choice. PEG 3350 is reported palatable and hence the compliance is excellent [14]. The only major drawback of this regimen is long preparation procedure time which led the parents to miss working days and absent school days for children. Hence raising the question if the duration of time could be minimised and still have same efficacy, safety, and tolerability of the medicine. Hence, one day PEG-3350 regimen was introduced and is studied with increased dose [17] [19]-[21] or combined with other laxatives [22]. The number of published studies investigating efficacy, safety and tolerability of one day PEG-3350 is relatively less. These studies were all single centered and varied widely in their design and only a few were prospective and randomized (Table 1) [17] [19]-[22]. This is the first review which tries to compile the studies so far on one day PEG-3350 without electrolyte as colonoscopy bowel preparation in children and attempts to summaries if this regimen can be commonly used in children for colonoscopy bowel preparation.

\section{Efficacy}

Efficacy of the bowel preparation is the clinical priority in high quality bowel preparation. The studies uses different outcome measures to define the success of the preparation 1 . The intubation success rate to cecal and terminal ileum 2. Non-standard bowel preparation rating Scale "excellent", "good", "fair", or "poor" 3. Boston Bowel Preparation Scale (BBPS). So far, one day PEG-3350 without electrolyte studies reported adequate and effective bowel preparation range from $77 \%-100 \%$ [17] [19] [21], cecum intubated range from $97 \%-100 \%$ [17] [19] and terminal ileum reached range from $84 \%$ - 100\% [19] [20]. Grading system graded by endoscopist in a prospective study showed excellent and good bowel preparation in 75\% [20]. Sorser et al. [21] in their prospective RCT comparing one day vs three days administering PEG-3350 without electrolyte showed no significant difference among the two groups with excellent and good $100 \%$ in one day vs $93 \%$ in three days. $77 \%$ of patients in a prospective study showed BBPS score of at least 5 [19]. When BBPS was compared between one day vs two days administration of PEG-3350 without electrolyte, there was no significant difference between excellent and good BBPS score of $70 \%$ in one day and $72 \%$ in two days [22].

\section{Safety}

All colonoscopy preparation are associated with adverse effects. Clinical adverse effects include nausea, vomiting, abdominal pain/cramping, bloating, fatigue, weakness, headache, and dizziness. In all the studies there are mild to moderate degree of above symptoms but none of these reported to have clinically significant need of intervention [13]-[19]. Interestingly, when the studies compared one day with two days [22] and one day with three days [21] administration PEG-3350 without electrolyte; nausea, vomiting, abdominal pain were comparatively same between both groups with no statistically significant difference between these groups.

Metabolic disturbance includes electrolytes imbalance and change in osmolarity. PEG-3350 preparation without electrolyte was commonly used with sports drinks. The mixture of these two when compared with PEG 
Table 1. One day PEG-3350 pediatrics colonoscopy preparation studies.

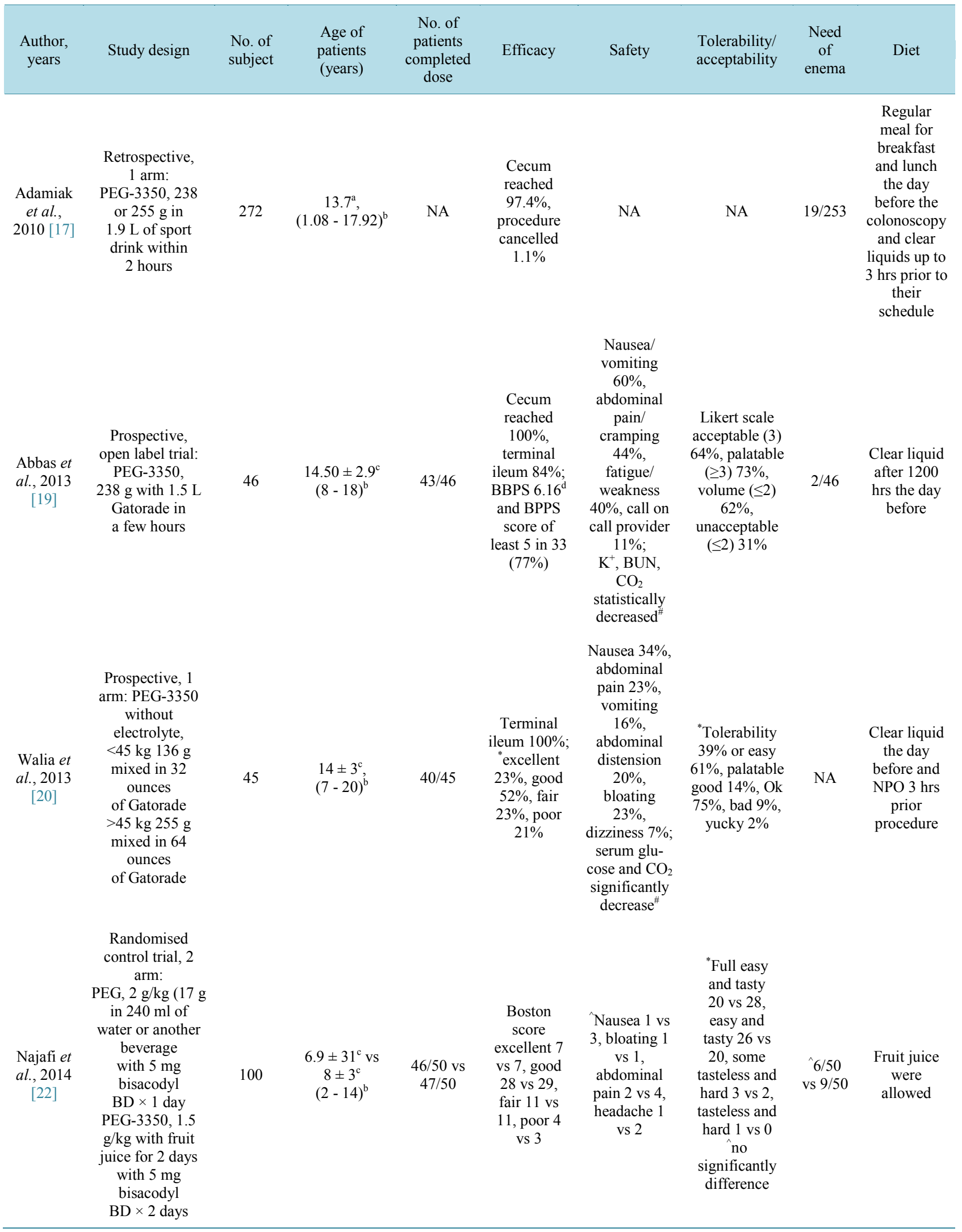




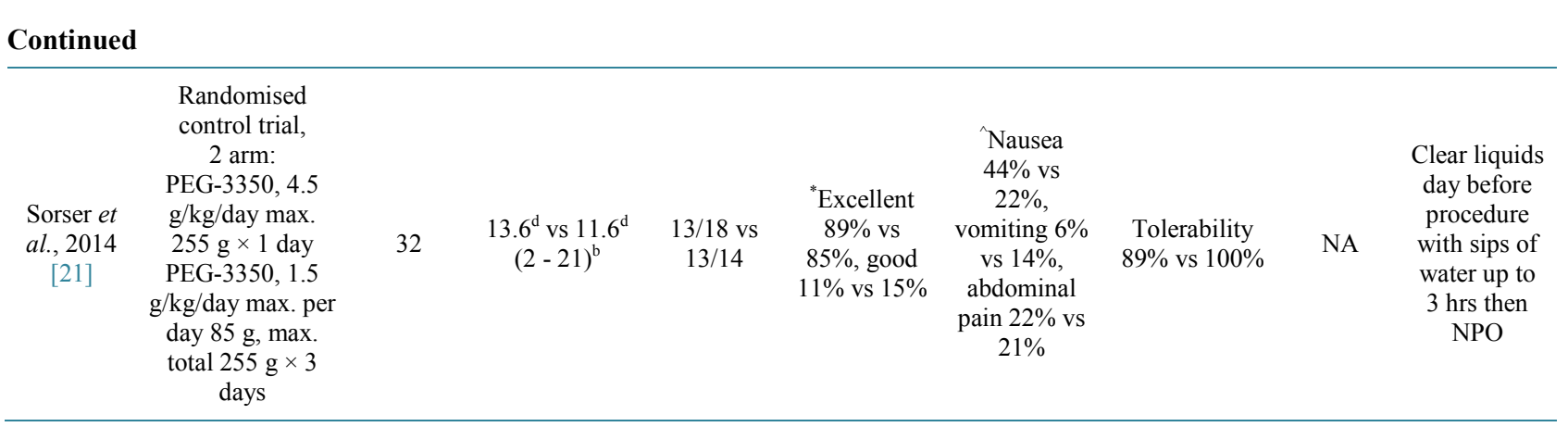

BBPS: Boston Bowel Preparation Scale, BUN: Blood urea nitrogen, max.: maximum, NPO: nil per oral, PEG: Polyethylene glycol. ${ }^{a}$ median, ${ }^{b}$ range, ${ }^{c}$ mean \pm $\mathrm{SD},{ }^{\mathrm{d}}$ mean. ${ }^{*}$ Non standard evaluation. ${ }^{*} \mathrm{p}<0.05 . \hat{\mathrm{p}}>0.05$. BPPS uses 10 point efficacy scale rating 0 - 3 in 3 section colon (right side, transverse side and left side) where $0=$ "unprepared colon due to solid stool", 1 = "portion of mucosa not seen", 2 = "minor amount of residual staining", and $3=$ "entire mucosa seen well with no staining". The sum of all 3 sections was added a total score from 0 to 9 . Likert scale from 1 to 5 with $1=$ "hated it", 2 = "didn't like it", $3=$ "Ok", 4 = "good" and 5 = "excellent".

with electrolyte (PEG-ELS) contained about 9 times less sodium, 4 times less potassium and 6 times less chloride [1]. Low sodium can lead to net absorption of free water resulting in hyponatremia [23], specially in patients with impaired kidney function. The carbohydrate in sports drinks may lead to bacterial fermentation and hence production of combustable gases [24]. However, till date there is no reported major adverse event of Gatorade mixed with PEG-3350 in the pediatrics literature [1] [25]. In a prospective studies, the post bowel preparation serum potassium, blood urea nitrogen (BUN), carbon dioxide were significantly low when compared to pre bowel preparation but these were reported clinically insignificant [19]. On the other hand, in other prospective study, electrolytes (sodium, potassium, chloride), BUN, creatinine has no significant pre and post procedure changes but had statistically significantly decrease in serum glucose and carbon dioxide [20]. Small changes in serum osmolarity was found in both one day and three days PEG-3350 without electrolyte administration but it was not clinically and statistically significant difference between both groups [21].

\section{Ease of Use}

PEG-3350 without electrolyte is palatable due to its tasteless character and can be mixed with any drink of patient choice. However, in order to decrease the duration of administration time, the volume of medicine has to be increased. Hence, one day PEG-3350 regimen has to be administered in large volume in limited period of time and therefore had unacceptable volume rating [19]. Despite this, when the children were asked if they would take this regimen again, all patients of a prospective study stated that they would like to take same bowel preparation again in future [20]. Among those patients, 9 patients had undergone colonoscopy bowel preparation in the past with alternate bowel preparation regimen. Unlike PEG-ELS, in PEG-3350 without electrolytes there is no such report of use of nasogastric tube for administration of the assigned amount.

\section{Quality of Evidence}

The major advantage of this regimen is its short duration over a few hours hence reducing preparation time, decreasing the working hours of parents and missed school days for children with same efficacy and safety as that of three-days and two-days PEG-3350 regimen. However, studies from which these conclusions are drawn are a small number of trials, which all have certain study bias. The data are collected from small sample size, tertiary centre, and the grading system they used to grade the efficacy of the bowel preparation is non-standardised. The study conducted by Adamiak et al. [17] is a retrospective study without proper controls, which cannot be compared to other preparation due to lack of investigation which was not done at the beginning (serum electrolytes). The author also mentions lack of standard dose of PEG-3350. Although the parents were advice to mixed specific dose of PEG-3350, it was not clear the exact amount of PEG-3350 consume by patients. Furthermore, it lacks standard scale to assess the bowel preparation quality. In other hand, even though Abbas et al. [19] used BBPS in their prospective study and Najafi et al. [22] in their RCT study to assess the bowel preparation quality, this scoring system has not been validated or previously used in children. In both prospective studies [17] [19] and RCT conducted by Sorser et al. [21] have mentioned there may be performance bias due to lack of blinding 
to the endoscopist during procedure. None of the study has reported the method of recruiting the patient hence having a selection bias as well.

\section{Conclusions}

One day PEG-3350 without electrolyte has been shown to be safe, effective with acceptable adverse effects but with low acceptance rate due to large volume that needs to be ingested in limited time.

So far the cumulative mean age of one day PEG-3350 is 12.5 years. Hence, there is less use of these regimen in younger children. Therefore, we cannot conclude if this regimen can be safely used among younger children.

The commercially available drinks that have been used to mix with PEG-3350, the components of electrolytes are not stated. Therefore, the true level of electrolyte in the mixture to be ingested is unknown and we suggest this should be noted in future studies.

Due to small number of studies conducted in this field we cannot precisely conclude that one day PEG-3350 can be routinely use on daily basis for colonoscopy bowel preparation in children. However, in emergency cases with limited time frame, who urgently needed colonoscopy and the patients who are unwilling to complete a longer duration preparation dose, this regimen could be considered. Physician should be vigilant and should consider adjusting the dose and duration of drug according to each child's condition. Further large, prospective, multi centre, high quality randomised control trial is needed.

\section{References}

[1] Hunter, A. and Mamula, P. (2010) Bowel Preparation for Pediatric Colonoscopy Procedures. Journal of Pediatrics Gastrointestinal and Nutrition, 51, 254-261. http://dx.doi.org/10.1097/MPG.0b013e3181eb6a1c

[2] Dahshan, A., Lin, C., Peters, J., Thomas, R. and Tolia, V. (1999) A Randomized, Prospective Study to Evaluated the Efficacy and Acceptance of Three Bowel Preparations for Colonoscopy in Children. American Journal of Gastrointestinal, 94, 3497-3501. http://dx.doi.org/10.1111/j.1572-0241.1999.01613.x

[3] da Silva, M.M., Briars, G.L., Patrick, M.K., Cleghorn, G.J. and Shepherd, R.W. (1997) Colonoscopy Preparation in Children: Safety, Efficacy, and Tolerance of High- versus Low-Volume Cleansing Methods. Journal of Gastroenterology and Nutrition, 24, 33-37. http://dx.doi.org/10.1097/00005176-199701000-00009

[4] Pinefield, A. and Stringer, M.D. (1999) Randomised Trail of Two Pharmacological Methods of Bowel Preparation for Day Case Colonoscopy. Archives of Disease on Childhood, 80, 181-183. http://dx.doi.org/10.1136/adc.80.2.181

[5] Tuner, D., Benchimol, E.I., Dunn, H., Griffiths, A.M., Frost, K., Scaini, V., Avolio, J. and Ling, S.C. (2009) Picosalax versus Polyethylene Glycol for Bowel Clean out before Colonoscopy in Children: A Randomized Controlled Trail. Endoscopy, 41, 1038-1045. http://dx.doi.org/10.1055/s-0029-1215333

[6] Safder, S., Demintieva, Y., Rewalt, M. and Elitsur, Y. (2008) Stool Consitency and Stool Frequency Are Excellent Clinical Markers for Adequate Colon Preparation after Polyethylene Glycol 3350 Cleansing Protocol: A Prospective Clinical Study in Children. Gastrointestinal Endoscopy, 68, 1131-1135. http://dx.doi.org/10.1016/j.gie.2008.04.026

[7] Schanz, S., Kruis, W., Mickisch, O., Kuppers, B., Berg, P., Frick, B., Heiland, G., Schenck, B., Horstkotte, H. and Winkler, A. (2008) Bowel Preparation for Colonoscopy with Sodium Phosphate Solution versus Polyethylene GlycolBased Lavage : A Multicenter Trail. Diagnostic and Therapeutic Endoscopy, 2008, Article ID: 713521. http://dx.doi.org/10.1155/2008/713521

[8] El-Baba, M.F., Padilla, M., Houstan, C., MAdani, S., Lin, C.H., Thomas, R. and Yolia, V. (2006) A Prospective Study Comparing Oral Sodium Phosphate Solution Phosphate Solution to a Bowel Cleansing Preparation with Nutrition Food Package in Children. Journal of Pediatric Gastroenterology and Nutrition, 42, 174-177. http://dx.doi.org/10.1097/01.mpg.0000189353.40419.31

[9] Gremse, D.A., Sacks, A.I. and Raines, S. (1996) Comparison of Oral Sodium Phosphate to Polyethylene Glycol-Based Solution for Bowel Preparation for Colonoscopy in Children. Journal of Pediatric Gastroenterolgy and Nutrition, 23, 586-590. http://dx.doi.org/10.1097/00005176-199612000-00013

[10] Ehrenpreis, E.D. (2009) Increased Serum Phosphate Levels and Calcium Fluxes Are Seen in Smaller Individuals after a Single Dose of Sodium Phosphate Colon Cleansing Solution: A Pharmacokinetic Analysis. Alimentary Pharmacology and Therapeutics, 29, 1201-1211. http://dx.doi.org/10.1111/j.1365-2036.2009.03987.x

[11] Shaoul, R., Wolff, R., Seligman, H., Tal, Y. and Jaffe, M. (2001) Symptoms of Hyperphosphatemia, Hypocalcemia, and Hypomagnesemia in an Adolescent Colonoscopy. Gastrointestinal Endoscopy, 53, 650-652. http://dx.doi.org/10.1067/mge.2001.112712

[12] Hasall, E. and Lobe, T.E. (2007) Risky Business: Oral Sodium Phosphate for Preparation Colonoscopy Bowel Prepara- 
tion in Children. Journal of Pediatric Gastroenterology and Nutrition, 45, 268-269. http://dx.doi.org/10.1097/MPG.0b013e318064c85e

[13] Zwas, F.R., Cirillo, N.W., El-Serag, H.B. and Eisen, R.N. (1996) Colonic Mucosal Abnormalities Associated with Oral Soduim Phosphate Solution. Gastrointestinal Endoscopy, 43, 463-466. http://dx.doi.org/10.1016/S0016-5107(96)70286-9

[14] Pashankar, D.S., Uc, A. and Bishop, W.P. (2004) Polyethylene Glycol 3350 without Electrolyte: A New Safe, Effective, and Palatable Bowel Preparation for Colonoscopy in Children. The Journal of Pediatric, 144, 358-362. http://dx.doi.org/10.1016/j.jpeds.2003.11.033

[15] Phatak, U.P., Johnson, S., Husain, S.Z. and Pashankar, D.S. (2011) Two-Day Bowel Preparation with Polyethylene Glycol 3350 and Bisacodyl: A New, Safe, and Effective Regimen for Colonoscopy in Children. Journal of Pediatric Gastrointestinal and Nutrition, 51, 71-74. http://dx.doi.org/10.1097/MPG.0b013e318210807a

[16] Kerkus, J., Horvath, A., Szychta, M., Woynarowski, M., Wegner, A., Wiernicka, A., Dadalski, M., Teisseyre, M. and Dziechciarz, P. (2013) High- versus Low-Volume Polyethylene Glycol plus Laxative versus Sennosides for Colonoscopy Preparation in Children. Journal of Pediatrics Gastrointestinal and Nutrition, 57, 230-235. http://dx.doi.org/10.1097/MPG.0b013e3182950ef5

[17] Adamiak, T., Altaf, M. and Jensen, M.K. (2010) One-Day Bowel Preparation Glycol 3350: An Effective Regimen for Colonoscopy in Children. Gastrointestinal Endoscopy, 71, 573-577. http://dx.doi.org/10.1016/j.gie.2009.10.042

[18] Jibaly, R., LaChance, J., Lecea, N., Ali, N. and Weber, J.E. (2011) The Utility of PEG3350 without Electrolytes for 2-Day Colonoscopy Preparation in Children. European Journal of Pediatric Surgery, 21, 318-321. http://dx.doi.org/10.1055/s-0031-1280822

[19] Abbas, M.I., Nylund, C.M., Bruch, C.J., Nazareno, L.G. and Rogers, P.L. (2013) Prospective Evaluation of 1-Day Polyethylene Glycol-3350 Bowel Preparation Regimen in Children. Journal of Pediatric Gastrointestinal and Nutrition, 56, 220-224. http://dx.doi.org/10.1097/MPG.0b013e31826630fc

[20] Walia, R., Steffen, R., Feinberg, L., Worley, S. and Mahajan, L. (2013) Tolerability, Safety, and Efficacy of PEG-3350 as a 1-Day Bowel Preparation in Children. Journal of Pediatric Gastrointestinal and Nutrition, 56, 225-228. http://dx.doi.org/10.1097/MPG.0b013e3182758c69

[21] Sorser, S.A., Konanki, V., Hursh, A., Hagglund, K. and Lyons, H. (2014) 1-Day Bowel Preparation with Polyethylene Glycol 3350 Is as Effective and Safe as a 3-Day Preparation for Colonoscopy in Children. BMC Research Notes, 15, 648. http://dx.doi.org/10.1186/1756-0500-7-648

[22] Najafi, M., Fallahi, G.H., Motamed, F., Farahmand, F., Khodadad, A., Ghajarzadeh, M., Rezaei, N. and Mehrabani, S. (2015) Comparision of One and Two-Day Bowel Preparation with Polyethylene Glycol in Pediatric Colonoscopy. Turkish Journal of Gastroenterology, 26, 232-235. http://dx.doi.org/10.5152/tjg.2015.6837

[23] Nagler, J., Poppers, D. and Turetz, M. (2006) Severe Hyponatremia and Seizure Following a Polyethylene GlycolBased Bowel Preparation for Colonoscopy. Journal of Clinical Gastroenterology, 40, 558-559. http://dx.doi.org/10.1097/00004836-200607000-00017

[24] Werner, S.D. (1996) Preoperative Preparation Prior to Colorectal Surgery. Gastrointestinal Endoscopy, 43, 530-531. http://dx.doi.org/10.1016/S0016-5107(96)70305-X

[25] Parakkal, D., Humberto, S., Muhammed, S. and Ehrenpreis, E.D. (2011) Preparing for Colonoscopy. In: Da Rocha, J.R., Ed., Endoscopic Procedures in Colon and Rectum, InTech, Winchester, 17-42. http://www.intechopen.com/books/endoscopic-procedures-in-colon-and-rectum/preparing-for-colonoscopy-2 